\title{
Center of the Goldman Lie algebra
}

\author{
ARPAN KABIRAJ
}

\begin{abstract}
We show that the center of the Goldman Lie algebra associated to a closed orientable surface is generated by the class of the trivial loop. For an orientable nonclosed surface of finite type, the center is generated by closed curves which are either homotopically trivial or homotopic to boundary components or punctures.
\end{abstract}

57M50; 57M07, 57M05

\section{Introduction}

Let $F$ be an oriented surface. Given two free homotopy classes of oriented closed curves $\alpha$ and $\beta$, consider two oriented closed curves $x$ and $y$ representing $\alpha$ and $\beta$, respectively. Performing a small homotopy if necessary, we can assume that $x$ and $y$ intersect transversally in double points. Goldman [10] defined the bracket of $\alpha$ and $\beta$ as the sum,

$$
[\alpha, \beta]=\sum_{p \in x \cap y} \iota(p)\langle x * p y\rangle
$$

where $x \cap y$ denotes the set of all intersection points between $x$ and $y, \iota(p)$ denotes the sign of the intersection between $x$ and $y$ at $p,(x * p y)$ denotes the loop product of $x$ and $y$ at $p$, and $\langle z\rangle$ denotes the free homotopy class of a curve $z$.

Let $\mathcal{C}$ be the set of all free homotopy classes of oriented closed curves in $F$. This bracket is extended by linearity to $\mathbb{Z}(\mathcal{C})$, the free module generated by $\mathcal{C}$. Goldman [10] showed that this bracket is well defined, skew-symmetric and satisfies the Jacobi identity. Therefore, this is a Lie bracket, and it gives a Lie algebra structure on $\mathbb{Z}(\mathcal{C})$, which we denote by $\mathcal{L}(F)$. Recall that the center of a Lie algebra $\mathcal{L}$ is the set of all elements $x$ in $\mathcal{L}$ such that $[x, y]=0$ for all $y$ in $\mathcal{L}$. The main object of this paper is to study the center of $\mathcal{L}(F)$.

The structure of the Goldman Lie algebra for surfaces of nonnegative Euler characteristic is either trivial or well understood; see Chas [3, Lemma 7.6] for the torus case.

Chas and Sullivan conjectured that, for a closed surface $F$, the center of the Goldman algebra is generated by the trivial loop. It is natural to conjecture (see Chas [4, 
Open Problem 1], [3, Problem 13.1] and Kawazumi and Kuno [12, Section 8.3]) that, for a surface $F$ with nonempty boundary, the center of $\mathcal{L}(F)$ is generated by the free homotopy classes of oriented closed curves which are either homotopic to a point, homotopic to a boundary, or homotopic to a puncture. In this paper, we prove these conjectures.

Main Theorem The center of the Goldman Lie algebra of any closed orientable surface $F$ is one-dimensional, and is generated by the class of the trivial loop. If $F$ is an orientable surface of finite type with boundary, then the center of $\mathcal{L}(F)$ is generated by the set of all free homotopy classes of oriented closed curves which are homotopic to either a point, a boundary component, or a puncture.

Remark 1 The closed case was done by Etingof in [8] using representation theory, but that proof did not address the case of surfaces with boundary. Our proof of both cases uses different ideas from hyperbolic geometry.

Goldman discovered this bracket while studying the Weil-Petersson symplectic form on Teichmüller spaces. Using Wolpert's [15] result on length and twist flow, he showed that if the Goldman bracket between two closed curves is zero and one of them has a simple representative, then their geometric intersection number is zero. The combinatorial structure of $\mathcal{L}(F)$ has also been studied. Using combinatorial topology and group theory, Chas [3] proved a stronger version of Goldman's result, namely if one of the curves has a simple representative, then the number of terms in the Goldman bracket is the same as their geometric intersection number. Chas and Krongold [6] proved that, for a compact surface with nonempty boundary, $\left[x, x^{3}\right]$ determines the self-intersection number of $x$. Using hyperbolic geometry, Chas and Gadgil [5] proved that there exists a positive integer $m_{0}$ such that, for all $m \geq m_{0}$, the geometric intersection number between $x$ and $y$ is the number of terms in $\left[x^{m}, y\right]$ divided by $m$. There is a Lie cobracket defined by Turaev [14] on $\mathbb{Z}(\mathcal{C})$ which is the dual object of the Goldman bracket. This structure has been studied by Chas and Krongold [7; 2].

Idea of the proof Our proof is based on hyperbolic geometry. Given an oriented surface of negative Euler characteristic, we fix a hyperbolic metric on it with geodesic boundary. There are two key ideas behind our proof.

The first idea is from [5]. Given two closed oriented curves $x$ and $y$ intersecting transversally, we construct lifts of $(x * p y)$ in the hyperbolic plane $\mathbb{H}$ for each intersection point $p$. By [5, Lemma 7.1], the lifts are quasigeodesics. Hence they are homotopic to unique geodesics. Therefore, if two terms $(x * p y)$ and $(x * q y)$ cancel each other, then the corresponding geodesics will be the same. By [5, Main Theorem], 
there exists $m_{0}$ such that if we take a power $m \geq m_{0}$ of $x$, then we can ensure that if the geodesics are the same, then the quasigeodesics are also the same, and hence the terms have the same sign. Therefore, there is no cancellation between the terms of $\left[x^{m}, y\right]$.

The second key idea is that all lifts of a simple closed geodesic are disjoint. Now, if an element $y=\sum_{i=1}^{k} y_{i}$ of $\mathcal{L}(F)$ belongs to the center, then we consider a simple closed curve $x$ which intersects at least one of the curves $y_{i}$ nontrivially. Taking sufficiently large powers of $x$ we can ensure that the same terms of $\left[x^{m}, y_{i}\right]$ have the same sign. Then, using that the lifts of $x$ are disjoint, we show that if one term of $\left[x^{m}, y_{i}\right]$ and another term of $\left[x^{m}, y_{j}\right]$ are the same, then $y_{i}$ and $y_{j}$ are conjugate.

Therefore, if $[x, y]$ is zero for all closed curves $x$, then each $y_{i}$ is disjoint from every simple closed curve, and hence each $y_{i}$ is either homotopic to a point or to a boundary component or to a puncture.

Organization of the paper Throughout the paper we follow the notation and definitions from [5].

In Section 2, we recall some basic facts about hyperbolic surfaces and closed curves on hyperbolic surfaces. We also mention a well-known result about hyperbolic elements of the fundamental group of a hyperbolic surface.

In Section 3, we recall from [5] the algebraic definition of the Goldman bracket between conjugacy classes of elements. Throughout the paper, we use this as the definition of Goldman bracket.

In Section 4, we recall from [5] the description of the lifts of the terms of Goldman bracket. We also state the lemma that these lifts are quasigeodesic and, therefore, in a neighborhood of a geodesic, following [5].

In Section 5, we show that if we take a sufficiently high power of a simple closed curve, then there is no cancellation between the terms of the Goldman bracket with any other closed curve.

In Section 6, we mention a classical theorem and prove the main theorems.

Acknowledgements The author would like to thank Siddhartha Gadgil for his encouragement and enlightening conversations. The author would like to thank Bidyut Sanki and Divakaran D. for all the discussions which helped him to understand the problem. The author would also like to thank Moira Chas for her valuable suggestions and comments. The author is supported by NET-CSIR (India) Senior Research Fellowship. 


\section{Closed curves on a hyperbolic surface}

In this section, we recall some basic facts about hyperbolic surfaces and closed curves on hyperbolic surfaces. We use the same notation as [5]. References for the results mentioned in this section are $[1 ; 5 ; 11 ; 13]$.

Let $F$ be an orientable surface of finite type with negative Euler characteristic; ie $F$ is a surface of genus $g$ with $b$ boundary components and $n$ punctures such that $2-2 g-b-n<0$. By [9, Theorem 1.2], we can endow $F$ with a hyperbolic metric. By a hyperbolic surface we mean an orientable surface with negative Euler characteristic and with a given hyperbolic metric. Given a hyperbolic surface $F$, we identify the fundamental group $\pi_{1}(F)$ of $F$ with a discrete subgroup of $\mathrm{PSL}_{2}(\mathbb{R})$, the group of orientation preserving isometries of the upper half plane $\mathbb{H}$. The action of $\pi_{1}(F)$ on $\mathbb{H}$ is properly discontinuous without fixed points, and the quotient space is isometric to $F$. Henceforth, by an isometry of $\mathbb{H}$ we mean an orientation preserving isometry, and by a closed curve we mean an oriented closed curve.

A homotopically nontrivial closed curve in $F$ is called essential if it is not homotopic to a puncture. A closed curve is called peripheral if it is homotopic to a power of a simple closed curve bounding a once-punctured disc. By a lift of a closed curve $\gamma$ to $\mathbb{H}$, we mean the image of a lift $\mathbb{R} \rightarrow \mathbb{H}$ of the map $\gamma \circ \pi$, where $\pi: \mathbb{R} \rightarrow S^{1}$ is the usual covering map.

There is a bijective correspondence between the set of all free homotopy classes of oriented closed curves in $F$ and the set of all conjugacy classes in $\pi_{1}(F)$ [5, Theorem 2.3]. Given an oriented closed curve $\gamma$ in $F$, we denote both its free homotopy class and the corresponding conjugacy class in $\pi_{1}(F)$ by $\langle\gamma\rangle$. Abusing notation, we sometimes denote the conjugacy class of $\gamma$ by $\gamma$ itself. Given $a, g \in \pi_{1}(F)$, we denote $g a g^{-1}$ by $a^{g}$ and the translation length of $a$ by $\tau_{a}$. If $a$ is hyperbolic, then $a^{g}$ is also hyperbolic with $\tau_{a^{g}}=\tau_{a}$ and $A_{a^{g}}=g A_{a}$ for all $g \in \pi_{1}(F)$, where $A_{a}$ denotes the axis of $a$.

The geometric intersection number between two free homotopy classes of closed curves $x$ and $y$, denoted by $i(x, y)$, is defined to be the minimal number of intersection points between a representative curve in the class $\langle x\rangle$ and a representative curve in the class $\langle y\rangle$ which intersect transversally in double points.

Every free homotopy class of an essential closed curve contains a unique closed geodesic whose length is the same as the translation length of any element of the corresponding conjugacy class. By a slight abuse of notation, we denote the free homotopy classes of essential closed curves by their geodesic representatives.

The following lemma is a well-known result. See [5, Corollary 6.3] for a proof. 
Lemma 2.1 Let $G$ be a discrete subgroup of $\mathrm{PSL}_{2}(\mathbb{R})$. Given two nonzero positive numbers $L$ and $C$, there exists a constant $M>0$ such that for every pair of hyperbolic elements $x$ and $y$ in $G$ with $\tau_{x} \leq L$ and $\tau_{y} \leq L$, the set

$$
\left\{x \in A_{x}: d\left(x, A_{y}\right)<C\right\}
$$

is either empty or a geodesic segment of length at most $M$.

\section{Goldman bracket}

In this section, we recall from [5] the algebraic definition of the Goldman bracket between two curves intersecting transversally (not necessarily in double points). For the equivalence of this definition with the previous one, see [5, Section 3].

Given two hyperbolic transformations $x$ and $y$ whose axes $A_{x}$ and $A_{y}$, respectively, intersect in a point $P$, let $I(x, y)$ denote the segment of $A_{x}$ of length $\tau_{x}$ starting from $P$ in the positive direction of $A_{x}$, containing $P$ but not containing $x P$.

Definition 3.1 Let $\langle x\rangle$ and $\langle y\rangle$ be two nontrivial conjugacy classes in $\pi_{1}(F)$. Define

$$
[\langle x\rangle,\langle y\rangle]=\left\{\begin{array}{cl}
\sum_{g Y \in J(x, y)} \iota\left(x, y^{g}\right)\left\langle x y^{g}\right\rangle & \text { if both } x \text { and } y \text { are hyperbolic, } \\
0 & \text { if either } x \text { or } y \text { is parabolic, }
\end{array}\right.
$$

where $Y$ is the cyclic subgroup generated by $y$,

$$
J(x, y)=\left\{g Y \in \pi_{1}(F) / Y: I(x, y) \cap g A_{y} \neq \varnothing\right\},
$$

and $\iota(x, y)$ denotes the sign of intersection between the axes of $x$ and $y$ if they intersect and 0 otherwise.

Remark 2 This definition is independent of the type of the intersection points of the representative curves. Therefore, we can use the geodesic representatives of the corresponding conjugacy classes (which intersect transversally but not necessarily in double points). Henceforth, we use this as the definition of the Goldman bracket.

\section{Terms of the Goldman bracket}

In this section we recall the description of the lifts of the terms of the Goldman bracket from [5, Section 7].

Let $x$ and $y$ be two hyperbolic elements in $\pi_{1}(F)$ whose axes intersect at the point $P$. Denote the projections of $A_{x}$ and $A_{y}$ in $F$ by $x_{1}$ and $y_{1}$ respectively. Let $p$ be the 


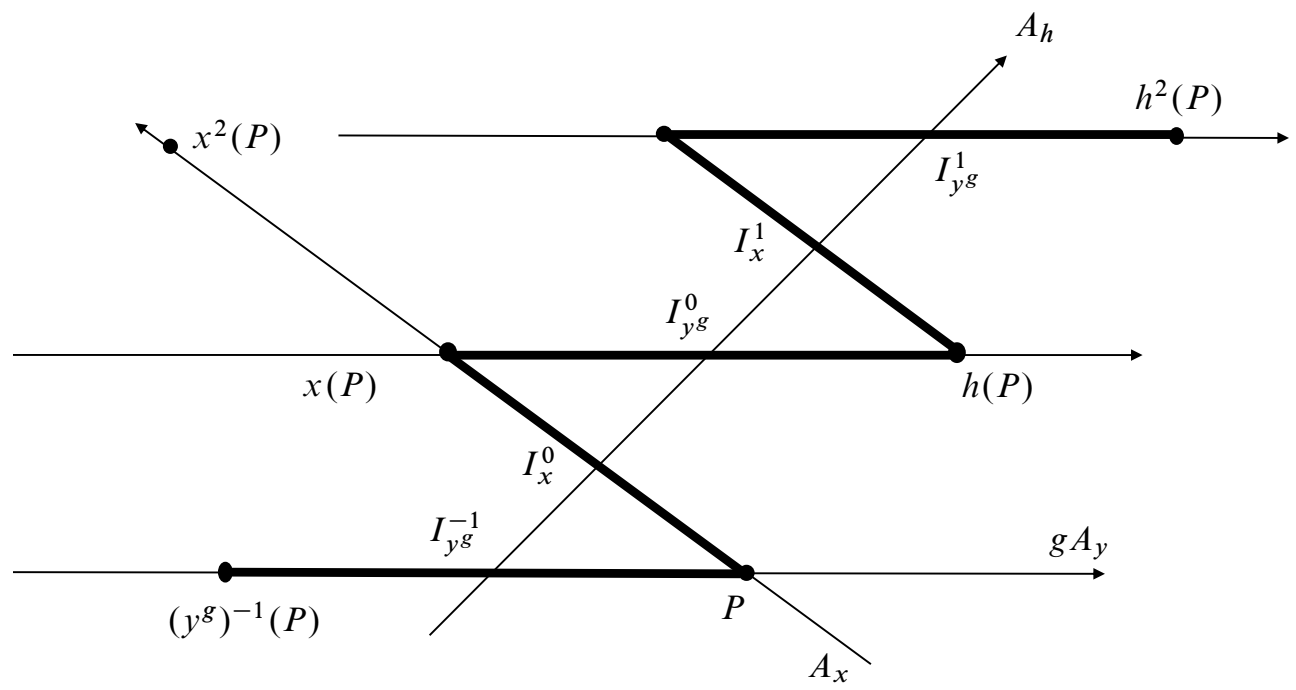

Figure 1: Lift of a term in the Goldman bracket

projection of $P$ in $F$. By [5, Remark 3.2], there exists a unique $g \in \pi_{1}(F)$ such that $g Y \in J(x, y)$ and $g Y$ corresponds to $p$. A lift of $\left(x_{1} *_{p} y_{1}\right)$ is a bi-infinite piecewise geodesic passing through $P$, which we denote by $\gamma\left(x, y^{g}\right)$; see Figure 1 .

Let $h=x y^{g}$. If we denote the geodesic arc from $P$ to $x(P)$ by $I_{x}^{0}$ and the geodesic segment from $x(P)$ to $h(P)$ by $I_{y g}^{0}$, then $\gamma\left(x, y^{g}\right)$ consists of geodesic segments of the form $h^{k}\left(I_{x}^{0}\right)$ and $h^{k}\left(I_{y^{g}}^{0}\right)$ occurring alternately.

Remark 3 Denote $h^{k}\left(I_{x}^{0}\right)$ by $I_{x}^{k}$ and $h^{k}\left(I_{y g}^{0}\right)$ by $I_{y g}^{k}$. From the definition, the length of $I_{x}^{k}$ is $\tau_{x}$ and the length of $I_{y g}^{k}$ is $\tau_{y}$ for all $k \in \mathbb{Z}$. Hence, by the description of the axis of the product of two isometries given in [5, Remark 3.4], $A_{h}$ intersects $I_{x}^{k}$ and $I_{y^{g}}^{k}$ in their midpoint for all $k \in \mathbb{Z}$.

For the definition of quasigeodesic and the proof of the following lemma, see [5, Section 7, Lemmas 7.1 and 7.2].

Lemma 4.1 Given $L>0$, there exist $K \geq 1$ and $C>0$, depending on $\pi_{1}(F)$, such that if $x$ and $y$ are two hyperbolic elements in $\pi_{1}(F)$ whose axes are distinct with $\tau_{x} \leq L$ and $\tau_{y} \leq L$, then for any $g \in \pi_{1}(F)$ and $m \in \mathbb{N}$, if $A_{x}$ and $A_{y^{g}}$ intersect, then:

(1) $\gamma\left(x^{m}, y^{g}\right)$ is a $K$-quasigeodesic, and $\gamma\left(x^{m}, y^{g}\right)$ is homotopic to $A_{h}$, where $h=x^{m} y^{g}$

(2) $\gamma\left(x^{m}, y^{g}\right) \subset N_{C / 2}\left(A_{h}\right)$ and $A_{h} \subset N_{C / 2}\left(\gamma\left(x^{m}, y^{g}\right)\right)$, where $N_{C}\left(A_{h}\right)$ denotes the $C$ neighborhood of $A_{h}$. 


\section{Noncancellation lemma}

Denote the length of a curve $x$ by $l(x)$. For the proof of the following lemma, see [5, Lemma 7.3].

Lemma 5.1 Let $L, K$ and $C$ be as in Lemma 4.1. For hyperbolic elements $x, y \in$ $\pi_{1}(F)$ with $\tau_{x} \leq L$ and $\tau_{y} \leq L$, let $m$ be a positive integer such that $m \tau_{x}>6 K C$.

Let $S$ and $R$ be the points in $I_{x^{m}}^{0}$ at distance $3 K C$ from $P$ and $x^{m} P$ (see Figure 2). Let $s$ (respectively $r$ ) be the open half-plane bounded by the line perpendicular to $A_{x}$ through $S$ (respectively $R$ ), containing the point $x^{m} P$ (respectively $P$ ).

Set $U=s \cap r \cap N_{C}\left(I_{x^{m}}^{0}\right)$. Then $U$ contains an open segment $J$ of $I_{x^{m}}^{0}$ such that $N_{C}\left(I_{x^{m}}^{0}\right) \backslash U$ is disconnected, $l(J) \geq m \tau_{x}-6 K C, U \subset N_{C}\left(I_{x^{m}}^{0}\right), \bar{U} \cap N_{C}\left(I_{x^{m}}^{k}\right)=\varnothing$ for all $k \neq 0$ and $\bar{U} \cap N_{C}\left(I_{y}^{k}\right)=\varnothing$ for all $k \in Z$.

The following lemma is the main lemma of this paper. The proof is based on the proof of [5, Lemma 7.4, Claims 1 and 2] and the idea that lifts of simple closed geodesics are disjoint.

Lemma 5.2 Let $x$ be a hyperbolic element in $\pi_{1}(F)$ such that the geodesic representative in the free homotopy class of $x$ is simple. Let $x_{1}=x^{h}$ for some $h \in \pi_{1}(F)$. Suppose $y$ and $y_{1}$ are two distinct hyperbolic elements in $\pi_{1}(F)$ whose axes are distinct and intersect the axes of $x$ and $x_{1}$, respectively. Let $L$ be a positive number such that the translation lengths of $x, y$ and $y_{1}$ are bounded above by $L$. Then there exists $m_{0}$ such that for any $m>m_{0}$, we have $\gamma\left(x^{m}, y\right)=\gamma\left(x_{1}^{m}, y_{1}\right)$ whenever $x^{m} y=x_{1}^{m} y_{1}$. Moreover, there exists $u \in \pi_{1}(F)$ such that $x_{1}=x^{u}$ and $y_{1}=y^{u}$.

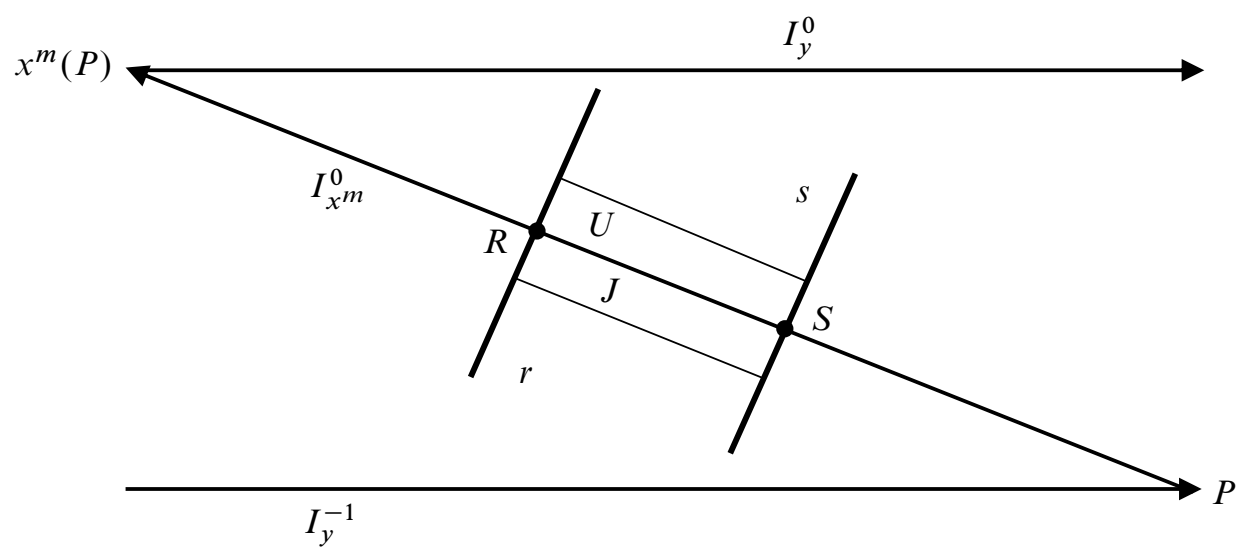

Figure 2: The open segment $J$ as in Lemma 5.2 
Proof Let $\tau_{0}$ be the systole, ie the length of a shortest length closed geodesic of $F$.

Let $C, K$ and $M$ be the constants defined in Lemmas 4.1 and 2.1 and let $m>m_{0}$. Define $m_{0}=K(3 M+10 C) / \tau_{0}$.

Since $x^{m} y=x_{1}^{m} y_{1}$, we have $A_{x^{m} y}=A_{x_{1}^{m} y_{1}}$. Let $g=x^{m} y=x_{1}^{m} y_{1}$. By Lemma 5.1,

$$
\gamma\left(x_{1}^{m}, y_{1}\right) \subset N_{C / 2}\left(A_{g}\right) \subset N_{C}\left(\gamma\left(x^{m}, y\right)\right) .
$$

Let $J$ and $U$ be as in Lemma 5.1 corresponding to $\gamma\left(x^{m}, y\right)$. Therefore, $J \subset U$, $J \subset I_{x^{m}}^{0} \subset \gamma\left(x^{m}, y\right)$, and length $(J) \geq m \tau_{x}-6 K C$.

Claim 1 The curve $\gamma\left(x_{1}^{m}, y_{1}\right)$ intersects $U$ and does not intersect the part of the boundary of $U$ contained in the boundary of $N_{C}\left(\gamma\left(x^{m}, y\right)\right)$.

Proof of claim If $\gamma\left(x_{1}^{m}, y_{1}\right)$ does not intersect $U$, by (1), $\gamma\left(x_{1}^{m}, y_{1}\right)$ is contained in $N_{C}\left(\gamma\left(x^{m}, y\right)\right) \backslash U=N_{C}\left(\gamma\left(x^{m}, y\right) \backslash J\right)$, which is disconnected. Hence by (1), $\gamma\left(x_{1}^{m}, y_{1}\right)$ should intersect both components, which contradicts that $\gamma\left(x_{1}^{m}, y_{1}\right)$ is connected. By Lemma 5.1, $\bar{U} \cap N_{C}\left(I_{x^{m}}^{k}\right)=\varnothing$ for all $k \neq 0$ and $\bar{U} \cap N_{C}\left(I_{y}^{k}\right)=\varnothing$ for all $k \in Z$. Therefore, $\gamma\left(x_{1}^{m}, y_{1}\right)$ does not intersect the part of the boundary of $U$ contained in the boundary of $N_{C}\left(\gamma\left(x^{m}, y\right)\right)$.

Therefore, any component of $U \cap \gamma\left(x_{1}^{m}, y_{1}\right)$ consists of piecewise geodesic arcs starting and ending at the sides of $U$ of length $2 C$.

Claim 2 Let $\beta$ be a component of $\gamma\left(x_{1}^{m}, y_{1}\right) \cap U$. Then $\beta$ contains a geodesic segment $l$ of length greater than $M$.

Proof of claim Case 1 Suppose $\beta$ contains more than three vertices. Then $\beta$ contains $I_{x_{1}^{m}}^{k}$ for some $k \in \mathbb{Z}$ and length $\left(I_{x_{1}^{m}}^{k}\right)=m \tau_{x_{1}}>m_{0} \tau_{0}=K(3 M+10 C)>M$.

Case 2 Suppose $\beta$ contains at most three vertices. Then $\beta$ consists of at most three segments. Let $v$ be the longest segment of $\beta$ and let $r=l(v)$. By hypothesis, $l(J) \geq m \tau_{x}-6 K C>3 K M+4 K C$. Using the triangle inequality and the properties of $m$ and $K$, we have

$$
(3 M+4 C) \leq K(3 M+4 C)<m \tau_{x}-6 K C \leq l(J) \leq 2 C+3 r+2 C=3 r+4 C .
$$

Hence $r>M$ which proves Claim 2.

The geodesic segment $v$ is contained in $\gamma\left(x_{1}^{m}, y_{1}\right)$ and $v \subset N_{C}\left(I_{x^{m}}^{0}\right)$. Therefore, by Lemma 2.1, $v$ intersects $I_{x^{m}}^{0}$ in a geodesic segment. Hence $I_{x^{m}}^{0}$ and $\gamma\left(x_{1}^{m}, y_{1}\right)$ intersect in a geodesic segment. 
Claim 3 If $\gamma\left(x^{m}, y\right)$ and $\gamma\left(x_{1}^{m}, y_{1}\right)$ intersect in a geodesic segment contained in $I_{x^{m}}^{0}$, then they are equal, and there exists $u \in \pi_{1}(F)$ such that $x_{1}=x^{u}$ and $y_{1}=y^{u}$.

Proof of claim As $I_{x^{m}}^{0}$ intersects $\gamma\left(x_{1}^{m}, y_{1}\right)$ in a geodesic segment, $I_{x^{m}}^{0}$ intersects either $I_{x_{1}^{m}}^{k}$ or $I_{y_{1}}^{k}$ in a geodesic segment.

Now $l\left(I_{x_{1}^{m}}^{k}\right)=m \tau_{x_{1}}=m \tau_{x}=l\left(I_{x^{m}}^{0}\right)$ for all $k \in \mathbb{Z}$, and $A_{g}$ intersects $I_{x^{m}}^{0}$ and $I_{x_{1}^{m}}^{k}$ in their midpoints. So if $I_{x^{m}}^{0}$ intersects $I_{x_{1}^{m}}^{k}$ in a geodesic segment, then they are equal.

If $I_{x^{m}}^{0}$ intersects $I_{y_{1}}^{k}$ in a geodesic segment for some $k \in \mathbb{Z}$, then by the construction of $\gamma\left(x^{m}, y\right)$, we see that $A_{x}$ intersects $I_{x_{1}^{m}}^{k+1}$, which lies in a translate of the geodesic $A_{x_{1}}$ and hence in a translate of $A_{x}$ (as $x$ and $x_{1}$ are conjugates). As the geodesic representative in the free homotopy class of $x$ is simple, all translates of $A_{x}$ are disjoint. Hence $I_{x^{m}}^{0}$ cannot intersect $I_{y_{1}}^{k}$ for any $k \in \mathbb{Z}$.

Since $I_{x^{m}}^{0}$ can not intersect $I_{y_{1}}^{k}$ in a geodesic segment, $I_{x^{m}}^{0}$ intersects $I_{x_{1}^{m}}^{k}$ for some $k \in \mathbb{Z}$. Thus $I_{x^{m}}^{0}=I_{x_{1}^{m}}^{k}$. Since $I_{y}^{0}$ and $I_{y_{1}}^{k}$ are the unique geodesic segments joining the end point of $I_{x^{m}}^{0}=I_{x_{1}^{m}}^{k}$ with the image of the starting point of $I_{x^{m}}^{0}=I_{x_{1}^{m}}^{k}$ under $g$, we see that $I_{y}^{0}=I_{y_{1}}^{k}$. By the periodic property of the definition of $\gamma\left(x^{m}, y\right)$ and $\gamma\left(x_{1}^{m}, y_{1}\right)$, they are equal. Since $g^{n} I_{x^{m}}^{0}=I_{x_{1}^{m}}^{0}$ and $g^{n} I_{y}^{0}=I_{y_{1}}^{0}$ for some $n$, taking $u=g^{n}$, we have $x_{1}=x^{u}$ and $y_{1}=y^{u}$. This proves the claim and thus the lemma.

\section{Center of the Goldman Lie algebra}

Lemma 6.1 Let $F$ be a hyperbolic surface. Suppose $x$ is an essential simple closed curve and $y$ is an essential closed curve. If $i(x, y) \neq 0$, then there exists $m_{0}$ such that $\left[x^{m}, y\right] \neq 0$ for all $m>m_{0}$.

Proof Let $L=\max \left\{\tau_{x}, \tau_{y}\right\}$ and $m_{0}$ be as in Lemma 5.2. If $m>m_{0}$, then

$$
\left[x^{m}, y\right]=m\left(\sum_{k B \in J\left(x^{m}, y\right)} \iota\left(x^{m}, y^{k}\right)\left\langle x^{m} y^{k}\right\rangle\right) .
$$

Suppose $\left\langle x^{m} y^{k}\right\rangle=\left\langle x^{m} y^{k_{1}}\right\rangle$. Then for some $g \in \pi_{1}(F)$,

$$
x^{m} y^{k}=\left(x^{m} y^{k_{1}}\right)^{g}=\left(x^{m}\right)^{g}(y)^{k_{1} g} .
$$

By Lemma 5.2, there exists $u \in \pi_{1}(F)$ such that $x$ is conjugate to $x^{g}$ and $y^{k}$ is conjugate to $y^{k_{1} g}$ by the element $u$. Therefore,

$$
\iota\left(x^{m}, y^{k}\right)=\iota\left(\left(x^{m}\right)^{u},\left(y^{k}\right)^{u}\right)=\iota\left(x^{m g}, y^{k_{1} g}\right)=\iota\left(x^{m}, y^{k_{1}}\right) .
$$

Hence $\left[x^{m}, y\right] \neq 0$. 
The following lemma is a classical result.

Lemma 6.2 Let $F$ be a hyperbolic surface of finite type with geodesic boundary. Let $\gamma$ be a closed curve whose geometric intersection number with any other nontrivial simple closed geodesic is zero. Then $\gamma$ is either homotopically trivial or homotopic to a boundary curve or peripheral.

Theorem 6.3 Let $F$ be a hyperbolic surface of finite type with geodesic boundary. Let $y=\sum_{i=1}^{n} c_{i} y_{i} \in \mathcal{L}(F)$, where each $y_{i}$ is a geodesic and $y_{i} \neq y_{j}$ for $i \neq j$. If $y$ belongs to the center of $\mathcal{L}(F)$, then $i\left(x, y_{i}\right)=\varnothing$ for every simple closed geodesic $x$ and for all $i \in\{1,2, \ldots, n\}$.

Proof We show that, given any simple closed geodesic $x$, if $i\left(x, y_{i}\right) \neq \varnothing$ for some $i \in\{1,2, \ldots, n\}$, then there exists $m \in \mathbb{N}$ such that $\left[x^{m}, y\right] \neq 0$.

Let $x$ be a simple closed geodesic which intersects at least one $y_{i}$. If some $y_{k}$ is disjoint from $x$, then the Goldman bracket between $x$ and $y_{k}$ is zero; therefore, without loss of generality, assume that $x$ intersects $y_{j}$ for all $j \in\{1,2, \ldots, n\}$. Let $L=\max \left\{\tau_{x}, \tau_{y_{1}}, \tau_{y_{2}}, \ldots, \tau_{y_{n}}\right\}$. Hence by Lemma 5.2, there exists $m_{i}$ for every $i \in\{1,2, \ldots, n\}$ such that, if $m>\max \left\{m_{i}\right\}$ with $x_{1}^{m}=\left(x^{m}\right)^{h}$ for some $h \in \pi_{1}(F)$, then $\gamma\left(x^{m}, y_{i}\right)=\gamma\left(x_{1}^{m}, y_{j}\right)$ whenever $x^{m} y_{i}=x_{1}^{m} y_{j}$. Also there exists $g \in \pi_{1}(F)$ such that $x_{1}=x^{g}$ and $y_{j}=y_{i}{ }^{g}$. Since $\left[x^{m}, \sum_{i=1}^{n} c_{i} y_{i}\right]=\sum_{i=1}^{n} c_{i}\left[x^{m}, y_{i}\right]$, by Lemma 6.1, it is enough to show that the terms of $\left[x^{m}, y_{i}\right]$ are distinct from the terms of $\left[x^{m}, y_{j}\right]$ for $i \neq j$.

Suppose $\left\langle x^{m} y_{i}^{k_{i}}\right\rangle=\left\langle x^{m} y_{j}^{k_{j}}\right\rangle$. Hence there exists $h \in \pi_{1}(F)$ such that

$$
x^{m} y_{i}^{k_{i}}=\left(x^{m} y_{j}^{k_{j}}\right)^{h}=\left(x^{m}\right)^{h}\left(y_{j}\right)^{k_{j} h} .
$$

By Lemma 5.2, $y_{i}^{k_{i}}$ and $y_{j}^{k_{j}}$ are conjugates of each other in $\pi_{1}(F)$. Therefore, $y_{i}$ and $y_{j}$ are freely homotopic to each other. Hence the geodesic representative corresponding to $y_{i}$ and $y_{j}$ are the same, which contradicts the assumption.

The Main Theorem follows at once from Lemma 6.2 and Theorem 6.3.

\section{References}

[1] A F Beardon, The geometry of discrete groups, Graduate Texts in Mathematics 91, Springer, New York (1983) MR

[2] M Chas, Combinatorial Lie bialgebras of curves on surfaces, Topology 43 (2004) 543-568 MR 
[3] M Chas, Minimal intersection of curves on surfaces, Geom. Dedicata 144 (2010) 25-60 MR

[4] M Chas, The Goldman bracket and the intersection of curves on surfaces, from "Geometry, groups and dynamics" (C S Aravinda, W M Goldman, K Gongopadhyay, A Lubotzky, M Mj, A Weaver, editors), Contemp. Math. 639, Amer. Math. Soc., Providence, RI (2015) 73-83 MR

[5] M Chas, S Gadgil, The Goldman bracket determines intersection numbers for surfaces and orbifolds, preprint (2012) arXiv

[6] M Chas, F Krongold, An algebraic characterization of simple closed curves on surfaces with boundary, J. Topol. Anal. 2 (2010) 395-417 MR

[7] M Chas, F Krongold, Algebraic characterization of simple closed curves via Turaev's cobracket, J. Topol. 9 (2016) 91-104 MR

[8] P Etingof, Casimirs of the Goldman Lie algebra of a closed surface, Int. Math. Res. Not. 2006 (2006) Art. ID 24894 MR

[9] B Farb, D Margalit, A primer on mapping class groups, Princeton Mathematical Series 49, Princeton Univ. Press (2012) MR

[10] W M Goldman, Invariant functions on Lie groups and Hamiltonian flows of surface group representations, Invent. Math. 85 (1986) 263-302 MR

[11] S Katok, Fuchsian groups, University of Chicago Press (1992) MR

[12] N Kawazumi, Y Kuno, The Goldman-Turaev Lie bialgebra and the Johnson homomorphisms, preprint (2013) arXiv

[13] J G Ratcliffe, Foundations of hyperbolic manifolds, 2nd edition, Graduate Texts in Mathematics 149, Springer, New York (2006) MR

[14] V G Turaev, Skein quantization of Poisson algebras of loops on surfaces, Ann. Sci. École Norm. Sup. 24 (1991) 635-704 MR

[15] S Wolpert, On the symplectic geometry of deformations of a hyperbolic surface, Ann. of Math. 117 (1983) 207-234 MR

Department of Mathematics, Indian Institute of Science

Bangalore 560012, India

arpan.into@math.iisc.ernet.in

Received: 19 February 2015 Revised: 13 December 2015 
